\title{
Assume-Guarantee Synthesis for Concurrent Reactive Programs with Partial Information ${ }^{\star}$
}

\author{
Roderick Bloem $^{1}$, Krishnendu Chatterjee ${ }^{2}$, Swen Jacobs ${ }^{1,3}$, and Robert Könighofer ${ }^{1}$ \\ ${ }^{1}$ IAIK, Graz University of Technology, Austria \\ ${ }^{2}$ IST Austria, Institute of Science and Technology, Austria \\ ${ }^{3}$ Reactive Systems Group, Saarland University, Germany
}

\begin{abstract}
Synthesis of program parts is particularly useful for concurrent systems. However, most approaches do not support common design tasks, like modifying a single process without having to re-synthesize or verify the whole system. Assume-guarantee synthesis (AGS) provides robustness against modifications of system parts, but thus far has been limited to the perfect information setting. This means that local variables cannot be hidden from other processes, which renders synthesis results cumbersome or even impossible to realize. We resolve this shortcoming by defining AGS under partial information. We analyze the complexity and decidability in different settings, showing that the problem has a high worstcase complexity and is undecidable in many interesting cases. Based on these observations, we present a pragmatic algorithm based on bounded synthesis, and demonstrate its practical applicability on several examples.
\end{abstract}

\section{Introduction}

Concurrent programs are notoriously hard to get right, due to unexpected behavior emerging from the interaction of different processes. At the same time, concurrency aspects such as mutual exclusion or deadlock freedom are easy to express declaratively. This makes concurrent programs an ideal subject for automatic synthesis. Due to the prohibitive complexity of synthesis tasks [33 34 17], the automated construction of entire programs from high-level specifications such as LTL is often unrealistic. More practical approaches are based on partially implemented programs that should be completed or refined automatically [17|1639], or program repair, where suitable replacements need to be synthesized for faulty program parts [25]. This paper focuses on such applications, where parts of the system are already given.

When several processes need to be synthesized or refined simultaneously, a fundamental question arises: What are the assumptions about the behavior of other processes on which a particular process should rely? The classical synthesis approaches assume either completely adversarial or cooperative behavior, which leads to problems in both

\footnotetext{
${ }^{\star}$ This work was supported by the Austrian Science Fund (FWF) through the research network RiSE (S11406-N23, S11407-N23) and grant nr. P23499-N23, by the European Commission through an ERC Start grant (279307: Graph Games) and project STANCE (317753), as well as by the German Research Foundation (DFG) through SFB/TR 14 AVACS and project ASDPS (JA 2357/2-1).
} 
cases: adversarial components may result in unrealizability of the system, while cooperative components may rely on a specific form of cooperation, and therefore are not robust against even small changes in a single process. Assume-Guarantee Synthesis (AGS) [9] uses a more reasonable assumption: processes are adversarial, but will not violate their own specification to obstruct others. Therefore, a system constructed by AGS will still satisfy its overall specification if we replace or refine one of the processes, as long as the new process satisfies its local specification. Furthermore, AGS leads to the desired solutions in cases where the classical notions (of cooperative or completely adversarial processes) do not, for example in the synthesis of mutual exclusion protocols [9] or fair-exchange protocols for digital contract signing [13].

A drawback of existing algorithms for AGS [9]13] is that they only work in a perfect information setting. This means that each component can access and use the values of all variables of the other processes. This is a major restriction, as most concurrent implementations rely on variables that are local to one process, and should not be changed or observed by the other process. While classical notions of synthesis have been considered in such partial information settings before [2817], we provide the first solution for AGS with partial information.

Contributions. In this work, we extend assume-guarantee synthesis to the synthesis of processes with partial information. In particular:

i) We analyze the complexity and decidability of AGS by reductions to games with three players. We distinguish synthesis problems based on informedness (perfect or partial) and resources (bounded or unbounded memory) of processes, and on specifications from different fragments of linear-time temporal logic (LTL).

ii) In light of the high complexity of many AGS problems, we propose a pragmatic approach, based on partially implemented programs and synthesis with bounded resources. We extend the bounded synthesis approach [18] to enable synthesis from partially defined, non-deterministic programs, and to the AGS setting.

iii) We provide the first implementation of AGS, integrated into a programming model that allows for a combined imperative-declarative programming style with finegrained, user-provided restrictions on the exchange of information between processes. To obtain efficient and simple code, our prototype also supports optimization of the synthesized program with respect to some basic user-defined metrics.

iv) We demonstrate the value of our approach on a number of small programs and protocols, including Peterson's mutual exclusion protocol, a double buffering protocol, and synthesis of atomic sections in a concurrent device driver. We also demonstrate how the robustness of AGS solutions allows us to refine parts of the synthesized program without starting synthesis from scratch.

\section{Motivating Example}

We illustrate our approach using the running example of [9], a version of Peterson's mutual exclusion protocol.

Sketch. We use the term sketch for concurrent reactive programs with non-deterministic choices. Listing 1 shows a sketch for Peterson's protocol with processes $P_{1}$ and $P_{2}$. 
Listing 1. Sketch of Peterson's mutual exclusion protocol. $\mathrm{F}=$ false, $\mathrm{T}=$ true.

0
1 cr $1:=\mathrm{F} ;$ wait $1:=\mathrm{F} ;$
2 do $\{$ // Process P1:
$3 \quad$ flag $1:=\mathrm{T} ;$
$4 \quad$ turn $:=\mathrm{T} ;$
$5 \quad$ while $\left(?_{1,1}\right) \quad\{\} / /$ wait $:=\mathrm{F} ;$ flag
$6 \quad \operatorname{cr} 1:=\mathrm{T} ;$
$7 \quad$ cr $1:=\mathrm{F} ;$ flag $1:=\mathrm{F} ;$ wait $1:=\mathrm{T} ;$
8 while $\left(? ?_{1,2}\right) \quad\{\} / /$ local work
9 wait $1:=\mathrm{F} ;$
$0\}$ while $(\mathrm{T})$

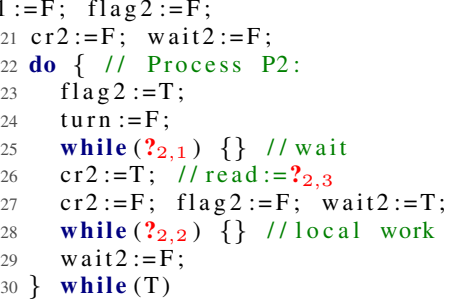

Variable flag $i$ indicates that $P_{i}$ wants to enter the critical section, and cri that $P_{i}$ is in the critical section. The first while-loop waits for permission to enter the critical section, the second loop models some local computation. Question marks denote non-deterministic choices, and we want to synthesize expressions that replace question marks such that $P_{1}$ and $P_{2}$ never visit the critical section simultaneously.

Specification. The desired properties of both processes are that (1) whenever a process wants to enter the critical section, it will eventually enter it (starvation freedom), and (2) the two processes are never in the critical section simultaneously (mutual exclusion). In LTL1 the specification is $\Phi_{i}=\mathrm{G}(\neg \mathrm{Cr} 1 \vee \neg \mathrm{Cr} 2) \wedge \mathrm{G}(\mathrm{flag} i \rightarrow \mathrm{F} \mathrm{cr} i)$, for $i \in\{1,2\}$.

Failure of Classical Approaches. There are essentially two options for applying standard synthesis techniques. First, we may assume that both processes are cooperative, and synthesize all $?_{i, j}$ simultaneously. However, the resulting implementation of $P_{2}$ may only work for the computed implementation of $P_{1}$, i.e., changing $P_{1}$ may break $P_{2}$. For instance, the solution $?_{1,1}=\operatorname{turn} \&$ flag2, $?_{2,1}=$ ! turn and $\boldsymbol{e}_{i, 2}=$ F satisfies the specification, but changing $?_{1,2}$ in $P_{1}$ to T will make $P_{2}$ starve. Note that this is not just a hypothetical case; we got exactly this solution in our experiments. As a second option, we may assume that the processes are adversarial, i.e., $P_{2}$ must work for any $P_{1}$ and vice versa. However, under this assumption, the problem is unrealizable [9].

Success of Assume-Guarantee Synthesis (AGS) [9]. AGS fixes this dilemma by requiring that $P_{2}$ must work for any realization of $P_{1}$ that satisfies its local specification (and vice versa). An AGS solution for Listing 1 is $?_{1,1}=\operatorname{turn} \& \mathrm{flag} 2, \boldsymbol{?}_{2,1}=$ ! turn \& flag2 and $?_{i, 2}=\mathrm{F}$ for $i \in\{1,2\}$.

Added Advantage of AGS. If one process in an AGS solution is changed or extended, but still satisfies its original specification, then the other, unchanged process is guaranteed to remain correct as well. We illustrate this feature by extending $P_{2}$ with a new variable named read. It is updated in a yet unknown way (expressed by $\boldsymbol{?}_{2,3}$ ) whenever $P_{2}$ enters the critical section in line 26 of Listing 1 . Assume that we want to implement $?_{2,3}$ such that read is true and false infinitely often. We take the solution from the previous paragraph and synthesize $?_{2,3}$ such that $P_{2}$ satisfies $\Phi_{2} \wedge(\mathrm{GF} \neg$ read $) \wedge($ G F read $)$, where $\Phi_{2}$ is the original specification of $P_{2}$. The fact that the modified process still

${ }^{1}$ In case the reader is not familiar with LTL: $\mathrm{G}$ is a temporal operator meaning "in all time steps"; likewise F means "at some point in the future". 
Listing 2. Result for Listing 1 turn is replaced by memory $\mathbf{m}$ in a clever way.

\begin{tabular}{|c|c|}
\hline 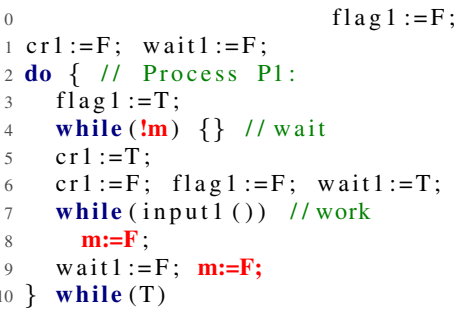 & \\
\hline
\end{tabular}

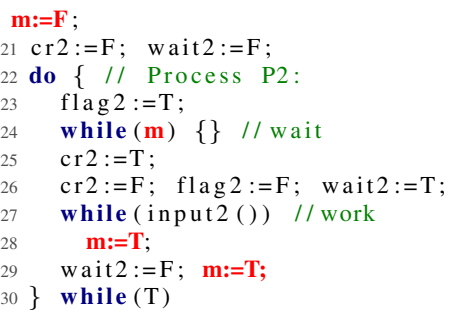

satisfies $\Phi_{2}$ implies that $P_{1}$ will still satisfy its original specification. We also notice that modular refinement saves overall synthesis time: our tool takes $19+55=74$ seconds to first synthesize the basic AGS solution for both processes and then refine $P_{2}$ in a second step to get the expected solution with $?_{2,3}=\neg$ read, while direct synthesis of the refined specification for both processes requires 263 seconds.

Drawbacks of the Existing AGS Framework [9]. While AGS provides important improvements over classical approaches, it may still produce solutions like $?_{1,1}=$ turn $\wedge \neg$ wait2 and $?_{2,1}=\neg$ turn $\wedge \neg$ wait1. However, wait2 is intended to be a local variable of $P_{2}$, and thus invisible for $P_{1}$. Solutions may also utilize modeling artifacts such as program counters, because AGS has no way to restrict the information visible to other processes. As a workaround, the existing approach [9] allows the user to define candidate implementations for each ?, and let the synthesis algorithm select one of the candidates. However, when implemented this way, a significant part of the problem needs to be solved by the user.

AGS with Partial Information. Our approach resolves this shortcoming by allowing the declaration of local variables. The user can write $f_{1,1}$ (turn, flag2) instead of $?_{1,1}$ to express that the solution may only depend on turn and flag2. Including more variables of $P_{1}$ does not make sense for this example, because their value is fixed at the call site. When setting $?_{\mathbf{2}, 1}=f_{1,2}(\operatorname{turn}, \mathrm{flag} 1)$ (and $?_{\mathbf{i}, 2}=f_{i, 2}()$ ), we get the solution proposed by Peterson: $?_{\mathbf{1}, \mathbf{1}}=\operatorname{turn} \wedge \mathrm{flag} 2$ and $?_{\mathbf{2}, 1}=\neg \operatorname{turn} \wedge \mathrm{flag} 1$ (and $?_{i, 2}=F$ ). This is the only AGS solution with these dependency constraints.

AGS with Additional Memory and Optimization. Our approach can also introduce additional memory in form of new variables. As with existing variables, the user can specify which question mark may depend on the memory variables, and also which variables may be used to update the memory. For our example, this feature can be used to synthesize the entire synchronization from scratch, without using turn, flag1, and $f l a g 2$. Suppose we remove turn, allow some memory $m$ instead, and impose the following restrictions: $?_{1,1}=f_{1,1}(\mathrm{flag} 2, \mathrm{~m}), ?_{2,1}=f_{2,1}(\mathrm{flag} 1, \mathrm{~m}), \boldsymbol{?}_{i, 2}$ is an uncontrollable input (to avoid overly simplistic solutions), and m can only be updated depending on the program counter and the old memory content. Our approach also supports cost functions over the result, and optimizes solutions iteratively. For our example, the user can assign costs for each memory update in order to obtain a simple solution with few memory updates. In this setup, our approach produces the solution 
presented in Listing 2. It is surprisingly simple: It requires only one bit of memory m, ignores both flags (although we did not force it to), and updates m only twice 2 . Our proof-of-concept implementation took only 74 seconds to find this solution.

\section{Definitions}

In this section we first define processes, refinement, schedulers, and specifications. Then we consider different versions of the co-synthesis problem, depending on informedness (partial or perfect), cooperation (cooperative, competitive, assume-guarantee), and resources (bounded or unbounded) of the players.

Variables, Valuations, Traces. Let $X$ be a finite set of binary variables. A valuation on $X$ is a function $v: X \rightarrow \mathbb{B}$ that assigns to each variable $x \in X$ a value $v(x) \in$ $\mathbb{B}$. We write $\mathbb{B}^{X}$ for the set of valuations on $X$, and $u \circ v$ for the concatenation of valuations $u \in \mathbb{B}^{X}$ and $v \in \mathbb{B}^{X^{\prime}}$ to a valuation in $\mathbb{B}^{X \cup X^{\prime}}$. A trace on $X$ is an infinite sequence $\left(v_{0}, v_{1}, \ldots\right)$ of valuations on $X$. Given a valuation $v \in \mathbb{B}^{X}$ and a subset $X^{\prime} \subseteq X$ of the variables, define $v \uparrow_{X^{\prime}}$ as the restriction of $v$ to $X^{\prime}$. Similarly, for a trace $\pi=\left(v_{0}, v_{1}, \ldots\right)$ on $X$, write $\pi \uparrow_{X^{\prime}}=\left(v_{0}\left\lceil X^{\prime}, v_{1}\left\lceil X^{\prime}, \ldots\right)\right.\right.$ for the restriction of $\pi$ to the variables $X^{\prime}$. The restriction operator extends naturally to sets of valuations and traces.

Processes and Refinement. We consider non-deterministic processes, where the nondeterminism is modeled by variables that are not under the control of the process. We call these variables input, but they may also be internal variables with non-deterministic updates. For $i \in\{1,2\}$, a process $P_{i}=\left(X_{i}, O_{i}, Y_{i}, \tau_{i}\right)$ consists of finite sets

- $X_{i}$ of modifiable state variables,

- $O_{i} \subseteq X_{3-i}$ of observable (but not modifiable) state variables,

- $Y_{i}$ of input variables,

and a transition function $\tau_{i}: \mathbb{B}^{X_{i}} \times \mathbb{B}^{O_{i}} \times \mathbb{B}^{Y_{i}} \rightarrow \mathbb{B}^{X_{i}}$. The transition function maps a current valuation of state and input variables to the next valuation for the state variables. We write $X=X_{1} \cup X_{2}$ for the set of state variables of both processes, and similarly $Y=Y_{1} \cup Y_{2}$ for the input variables. Note that some variables may be shared by both processes. Variables that are not shared between processes will be called local variables.

We obtain a refinement of a process by resolving some of the non-determinism introduced by input variables, and possibly extending the sets of local state variables. Formally, let $C_{i} \subseteq Y_{i}$ be a set of controllable variables, let $Y_{i}^{\prime}=Y_{i} \backslash C_{i}$, and let $X_{i}^{\prime} \supseteq X_{i}$ be an extended (finite) set of state variables, with $X_{1}^{\prime} \cap X_{2}^{\prime}=X_{1} \cap X_{2}$. Then a refinement of process $P_{i}=\left(X_{i}, O_{i}, Y_{i}, \tau_{i}\right)$ with respect to $C_{i}$ is a process $P_{i}^{\prime}=\left(X_{i}^{\prime}, O_{i}, Y_{i}^{\prime}, \tau_{i}^{\prime}\right)$ with a transition function $\tau_{i}^{\prime}: \mathbb{B}^{X_{i}^{\prime}} \times \mathbb{B}^{O_{i}} \times \mathbb{B}^{Y_{i}^{\prime}} \rightarrow \mathbb{B}^{X_{i}^{\prime}}$ such that for all $\bar{x} \in \mathbb{B}^{X_{i}^{\prime}}, \bar{o} \in \mathbb{B}^{O_{i}}, \bar{y} \in \mathbb{B}^{Y_{i}^{\prime}}$ there exists $\bar{c} \in \mathbb{B}^{C_{i}}$ with

$$
\left.\left.\tau_{i}^{\prime}(\bar{x}, \bar{o}, \bar{y})\right|_{X_{i}}=\tau_{i}(\bar{x}\rceil_{X_{i}}, \bar{o}, \bar{y} \circ \bar{c}\right) .
$$

We write $P_{i}^{\prime} \preceq P_{i}$ to denote that $P_{i}^{\prime}$ is a refinement of $P_{i}$.

\footnotetext{
${ }^{2}$ The memory $\mathrm{m}$ is updated whenever an input is read in line 7 or 27 we copied the update into both branches to increase readability.
} 
Important Modeling Aspects. Local variables are used to model partial information: all decisions of a process need to be independent of the variables that are local to the other process. Furthermore, variables in $X_{i}^{\prime} \backslash X_{i}$ are used to model additional memory that a process can use to store observed information. We say a refinement is memoryless if $X_{i}^{\prime}=X_{i}$, and it is b-bounded if $\left|X_{i}^{\prime} \backslash X_{i}\right| \leq b$.

Schedulers, Executions. A scheduler for processes $P_{1}$ and $P_{2}$ chooses at each computation step whether $P_{1}$ or $P_{2}$ can take a step to update its variables. Let $\mathcal{X}_{1}, \mathcal{X}_{2}$ be the sets of all variables (state, memory, input) of $P_{1}$ and $P_{2}$, respectively, and let $\mathcal{X}=\mathcal{X}_{1} \cup \mathcal{X}_{2}$. Let furthermore $V=\mathbb{B}^{\mathcal{X}}$ be the set of global valuations. Then, the scheduler is a function sched : $V^{*} \rightarrow\{1,2\}$ that maps a finite sequence of global valuations to a process index $i \in\{1,2\}$. Scheduler sched is fair if for all traces $\left(v_{0}, v_{1}, \ldots\right) \in V^{\omega}$ it assigns infinitely many turns to both $P_{1}$ and $P_{2}$, i.e., there are infinitely many $j \geq 0$ such that $\operatorname{sched}\left(v_{0}, \ldots, v_{j}\right)=1$, and infinitely many $k \geq 0 \operatorname{such}$ that $\operatorname{sched}\left(v_{0}, \ldots, v_{k}\right)=2$.

Given two processes $P_{1}, P_{2}$, a scheduler sched, and a start valuation $v_{0}$, the set of possible executions of the parallel composition $P_{1}\left\|P_{2}\right\|$ sched is

$$
\llbracket P_{1}\left\|P_{2}\right\| \text { sched, } v_{0} \rrbracket=\left\{\begin{array}{l|l}
\left(v_{0}, v_{1}, \ldots\right) \in V^{\omega} & \begin{array}{l}
\forall j \geq 0 . \operatorname{sched}\left(v_{0}, v_{1}, \ldots, v_{j}\right)=i \\
\text { and } v_{j+1}\left\lceil\left(\mathcal{X} \backslash \mathcal{X}_{i}\right)=v_{j}\left\lceil\left(\mathcal{X} \backslash \mathcal{X}_{i}\right)\right.\right. \\
\text { and } v_{j+1}\left\lceil\mathcal { X } _ { i } \backslash Y _ { i } \in \tau _ { i } \left( v_{j}\left\lceil\mathcal{X}_{i}\right)\right.\right.
\end{array}
\end{array}\right\} .
$$

That is, at every turn the scheduler decides which of the processes makes a transition, and the state and memory variables are updated according to the transition function of that process. Note that during turns of process $P_{i}$, the values of local variables of the other process (in $\mathcal{X} \backslash \mathcal{X}_{i}$ ) remain unchanged.

Safety, GR(1), LTL. A specification $\Phi$ is a set of traces on $X \cup Y$. We consider $\omega$ regular specifications, in particular the following fragments of LTL 3

- safety properties are of the form $\mathrm{G} B$, where $B$ is a Boolean formula over variables in $X \cup Y$, defining a subset of valuations that are safe.

- GR(1) properties are of the form $\left(\bigwedge_{i} \mathrm{GF} L_{e}^{i}\right) \rightarrow\left(\bigwedge_{j} \mathrm{GF} L_{s}^{j}\right)$, where the $L_{e}^{i}$ and $L_{s}^{j}$ are Boolean formulas over $X \cup Y$.

- LTL properties are given as arbitrary LTL formulas over $X \cup Y$. They are a subset of the $\omega$-regular properties.

Co-Synthesis. In all co-synthesis problems, the input to the problem is given as: two processes $P_{1}, P_{2}$ with $P_{i}=\left(X_{i}, O_{i}, Y_{i}, \tau_{i}\right)$, two sets $C_{1}, C_{2}$ of controllable variables with $C_{i} \subseteq Y_{i}$, two specifications $\Phi_{1}, \Phi_{2}$, and a start valuation $v_{0} \in \mathbb{B}^{X \cup Y}$, where $Y=Y_{1} \cup Y_{2}$.

Cooperative co-synthesis. The cooperative co-synthesis problem is to find out whether there exist two processes $P_{1}^{\prime} \preceq P_{1}$ and $P_{2}^{\prime} \preceq P_{2}$, and a valuation $v_{0}^{\prime}$ with $\left.v_{0}^{\prime}\right|_{X \cup Y}=v_{0}$, such that for all fair schedulers sched we have

$$
\llbracket P_{1}^{\prime}\left\|P_{2}^{\prime}\right\| \text { sched, } v_{0}^{\prime} \rrbracket\left\lceil X \cup Y \subseteq \Phi_{1} \wedge \Phi_{2} .\right.
$$

\footnotetext{
${ }^{3}$ For a definition of syntax and semantics of LTL, see e.g. [15].
} 
Competitive co-synthesis. The competitive co-synthesis problem is to determine whether there exist two processes $P_{1}^{\prime} \preceq P_{1}$ and $P_{2}^{\prime} \preceq P_{2}$, and a valuation $v_{0}^{\prime}$ with $\left.v_{0}^{\prime}\right|_{X \cup Y}=v_{0}$, such that for all fair schedulers sched we have

(i) $\llbracket P_{1}^{\prime}\left\|P_{2}\right\|$ sched, $v_{0}^{\prime} \rrbracket\left\lceil X \cup Y \subseteq \Phi_{1}\right.$, and

(ii) $\llbracket P_{1}\left\|P_{2}^{\prime}\right\|$ sched, $v_{0}^{\prime} \rrbracket \mid X \cup Y \subseteq \Phi_{2}$.

Assume-guarantee Synthesis. The assume-guarantee synthesis (AGS) problem is to determine whether there exist two processes $P_{1}^{\prime} \preceq P_{1}$ and $P_{2}^{\prime} \preceq P_{2}$, and a valuation $v_{0}^{\prime}$ with $v_{0}^{\prime} \uparrow_{X \cup Y}=v_{0}$, such that for all fair schedulers sched we have

(i) $\llbracket P_{1}^{\prime}\left\|P_{2}\right\|$ sched, $v_{0}^{\prime} \rrbracket\left\lceil X \cup Y \subseteq \Phi_{2} \rightarrow \Phi_{1}\right.$,

(ii) $\llbracket P_{1}\left\|P_{2}^{\prime}\right\|$ sched, $v_{0}^{\prime} \rrbracket\left\lceil X \cup Y \subseteq \Phi_{1} \rightarrow \Phi_{2}\right.$, and

(iii) $\llbracket P_{1}^{\prime}\left\|P_{2}^{\prime}\right\|$ sched, $v_{0}^{\prime} \rrbracket \mid X \cup Y \subseteq \Phi_{1} \wedge \Phi_{2}$.

We refer the reader to [9] for more intuition and a detailed discussion of AGS.

Informedness and Boundedness. A synthesis problem is under perfect information if $X_{i} \cup O_{i}=X$ for $i \in\{1,2\}$, and $Y_{1}=Y_{2}$. That is, both processes have knowledge about all variables in the system. Otherwise, it is under partial information. A synthesis problem is memoryless (or $b$-bounded) if we additionally require that $P_{1}^{\prime}, P_{2}^{\prime}$ are memoryless (or $b$-bounded) refinements of $P_{1}, P_{2}$.

Optimization Criteria. Let $\mathcal{P}$ be the set of all processes. A cost function is a function cost : $\mathcal{P} \times \mathcal{P} \rightarrow \mathbb{N}$ that assigns a cost to a tuple of processes. By requiring that the cost of solutions is minimal or below a certain threshold, we will use cost functions to optimize synthesis results.

Note on Robustness against Modifications. Suppose $P_{1}^{\prime}, P_{2}^{\prime}$ are the result of AGS on a given input, including specifications $\Phi_{1}, \Phi_{2}$. By the properties of AGS, this solution is robust against replacing one of the processes, say $P_{2}$, with a different solution: if a replacement $P_{2}^{\prime \prime}$ of $P_{2}^{\prime}$ satisfies $\Phi_{2}$, then the overall system will still be correct. If we furthermore ensure that conditions (ii) and (iii) of AGS are satisfied by $P_{1}^{\prime}$ and $P_{2}^{\prime \prime}$, then this pair is again an AGS solution, i.e., we can go on and refine another process.

Co-synthesis of more than 2 Processes. The definitions above naturally extend to programs with more than 2 concurrent processes, cp. [13] for AGS with 3 processes.

\section{Complexity and Decidability of AGS}

We give an an overview of the complexity of AGS. The complexity results are with respect to the size of the input, where the input consists of the given non-deterministic state transition system and the specification formula (i.e., the size of the input is the size of the explicit state transition system and the length of the formula).

Theorem 1. The complexity of AGS is given in the following table:

\begin{tabular}{|l|c|c|c|c|}
\hline & \multicolumn{2}{|c|}{ Bounded Memory } & \multicolumn{2}{c|}{ Unbounded Memory } \\
\hline & Perfect Inf. & Partial Inf. & Perfect Inf. & Partial Inf. \\
\hline Safety & $P$ & NP-C & $P$ & Undec \\
GR(1) & NP-C & NP-C & $P$ & Undec \\
LTL & PSPACE-C & PSPACE-C & 2EXP-C & Undec \\
\hline
\end{tabular}


Note that the complexity classes for memoryless AGS are the same as for AGS with bounded memory - the case of bounded memory reduces to the memoryless case, by considering a game that is larger by a constant factor: the given bound.

Also note that if we consider the results in the order given by the columns of the table, they form a non-monotonic pattern: (1) For safety objectives the complexity increases and then decreases (from PTIME to NP-complete to PTIME again); (2) for GR(1) objectives it remains NP-complete and finally decreases to PTIME; and (3) for LTL it remains PSPACE-complete and then increases to 2 EXPTIME-complete.

In the following, we give proof ideas for these complexity results. For formal definitions of three-player games, we refer the reader to [9].

\section{Proof Ideas}

\section{First Column: Bounded Memory, Perfect Information}

Safety: It was shown in [9] that AGS solutions can be obtained from the solutions of games with secure equilibria. It follows from the results of [10] that for games with safety objectives, the solution for secure equilibria reduces to solving games with safety and reachability objectives for which memoryless strategies suffice (i.e., memoryless strategies are as powerful as arbitrary strategies for safety objectives). It also follows from [10] that for safety objectives, games with secure equilibria can be solved in polynomial time.

$G R(1)$ : It follows from the results of [20] that even in a graph (not a game) the question whether there exists a memoryless strategy to visit two distinct states infinitely often is NP-hard (a reduction from directed subgraph homeomorphism). Since visiting two distinct states infinitely often is a conjunction of two Büchi objectives, which is a special case of GR(1) objectives, the lower bound follows. For the NP upper bound, the witness memoryless strategy can be guessed, and once a memoryless strategy is fixed, we have a graph, and the polynomial-time verification procedure is the polynomial-time algorithm for model checking graphs with GR(1) objectives [32].

LTL: In the special case of a game graph where every player-1 state has exactly one outgoing edge, the memoryless AGS problem is an LTL model checking problem, and thus the lower bound of LTL model checking [15] implies PSPACE-hardness. For the upper bound, we guess a memoryless strategy (as for GR(1)), and the verification problem is an LTL model checking question. Since LTL model checking is in PSPACE [15] and NPSPACE=PSPACE (by Savitch's theorem) [37|30], we obtain the desired result.

\section{Second Column: Bounded Memory, Partial Information}

Safety: The lower bound result was established in [12]. For the upper bound, again the witness is a memoryless strategy. Given the fixed strategy, we have a graph problem with safety and reachability objectives that can be solved in polynomial time (for the polynomial-time verification).

$G R(1)$ : The lower bound follows from the perfect-information case; for the upper bound, we can again guess and check a memoryless strategy.

LTL: Similar to the perfect information case, given above. 


\section{Third Column: Unbounded Memory, Perfect Information}

Safety: As mentioned before, for AGS under perfect information and safety objectives, the memoryless and the general problem coincide, implying this result.

$G R(1)$ : It follows from results of [9]10] that solving AGS for perfect-information games requires solving games with implication conditions. Since games with implication of GR(1) objectives can be solved in polynomial time [21], the result follows.

LTL: The lower bound follows from standard LTL synthesis [33]. For the upper bound, AGS for perfect-information games requires solving implication games, and games with implication of LTL objectives can be solved in 2EXPTIME [33]. The desired result follows.

\section{Fourth Column: Unbounded Memory, Partial Information}

It was shown in [31] that three-player partial-observation games are undecidable, and it was also shown that the undecidability result holds for safety objectives too [11].

\section{Algorithms for AGS}

Given the undecidability of AGS in general, and its high complexity for most other cases, we propose a pragmatic approach that divides the general synthesis problem into a sequence of synthesis problems with a bounded amount of memory, and encodes the resulting problems into SMT formulas. Our encoding is inspired by the Bounded Synthesis approach [18], but supports synthesis from non-deterministic program sketches, as well as AGS problems. By iteratively deciding whether there exists an implementation for an increasing bound on the number of memory variables, we obtain a semidecision procedure for AGS with partial information.

We first define the procedure for cooperative co-synthesis problems, and then show how to extend it to AGS problems.

\subsection{SMT-Based Co-synthesis from Program Sketches}

Consider a cooperative co-synthesis problem with inputs $P_{1}$ and $P_{2}$, defined as $P_{i}=$ $\left(X_{i}, O_{i}, Y_{i}, \tau_{i}\right)$, two sets $C_{1}, C_{2}$ of controllable variables with $C_{i} \subseteq Y_{i}$, a specification $\Phi_{1} \wedge \Phi_{2}$, and a start valuation $v_{0} \in \mathbb{B}^{X \cup Y}$, where $Y=Y_{1} \cup Y_{2}$.

In the following, we describe a set of SMT constraints such that a model represents refinements $P_{1}^{\prime} \preceq P_{1}, P_{2}^{\prime} \preceq P_{2}$ such that for all fair schedulers sched, we have $\llbracket P_{1}^{\prime} \|$ $P_{2}^{\prime} \|$ sched, $v_{0} \rrbracket \subseteq \Phi_{1} \wedge \Phi_{2}$. Assume we are given a bound $b \in \mathbb{N}$, and let $Z_{1}, Z_{2}$ be disjoint sets of additional memory variables with $\left|Z_{i}\right|=b$ for $i \in\{1,2\}$.

Constraints on given Transition Functions. In the expected way, the transition functions $\tau_{1}$ and $\tau_{2}$ are declared as functions $\tau_{i}: \mathbb{B}^{X_{i}} \times \mathbb{B}^{O_{i}} \times \mathbb{B}^{Y_{i}} \rightarrow \mathbb{B}^{X_{i}}$, and directly encoded into SMT constraints by stating $\tau_{i}(\bar{x}, \bar{o}, \bar{y})=\bar{x}^{\prime}$ for every $\bar{x} \in \mathbb{B}^{X_{i}}, \bar{o} \in$ $\mathbb{B}^{O_{i}}, \bar{y} \in \mathbb{B}^{Y_{i}}$, according to the given transition functions $\tau_{1}, \tau_{2}$.

Constraints for Interleaving Semantics, Fair Scheduling. To obtain an encoding for interleaving semantics, we add a scheduling variable $s$ to both sets of inputs $Y_{1}$ and $Y_{2}$, and require that (i) $\tau_{1}(\bar{x}, \bar{o}, \bar{y})=\bar{x}$ whenever $\bar{y}(s)=$ false, and (ii) $\tau_{2}(\bar{x}, \bar{o}, \bar{y})=$ 
$\bar{x}$ whenever $\bar{y}(s)=$ true. Fairness of the scheduler can then be encoded as the LTL formula $\mathrm{GF} s \wedge \mathrm{GF} \neg s$, abbreviated fair in the following.

Constraints on Resulting Strategy. Let $X_{i}^{\prime}=X_{i} \cup Z_{i}$ be the extended state set, and $Y_{i}^{\prime}=Y_{i} \backslash C_{i}$ the set of input variables of process $P_{i}^{\prime}$, reduced by its controllable variables. Then the resulting strategy of $P_{i}^{\prime}$ is represented by functions $\mu_{i}: \mathbb{B}^{X_{i}^{\prime}} \times$ $\mathbb{B}^{O_{i}} \times \mathbb{B}^{Y_{i}^{\prime}} \rightarrow \mathbb{B}^{Z_{i}}$ to update the memory variables, and $f_{i}: \mathbb{B}^{X_{i}^{\prime}} \times \mathbb{B}^{O_{i}} \times \mathbb{B}^{Y_{i}^{\prime}} \rightarrow \mathbb{B}^{C_{i}}$ to resolve the non-determinism for controllable variables. Functions $f_{i}$ and $\mu_{i}$ for $i \in$ $\{1,2\}$ are constrained indirectly using constraints on an auxiliary annotation function that will ensure that the resulting strategy satisfies the specification $\Phi=\left(\right.$ fair $\rightarrow \Phi_{1} \wedge$ $\left.\Phi_{2}\right)$. To obtain these constraints, first transform $\Phi$ into a universal co-Büchi automaton $\mathcal{U}_{\Phi}=\left(Q, q_{0}, \Delta, F\right)$, where

- $Q$ is a set of states and $q_{0} \in Q$ is the initial state,

- $\Delta \subseteq Q \times Q$ is a set of transitions, labeled with valuations $v \in \mathbb{B}^{X_{1} \cup X_{2} \cup Y_{1} \cup Y_{2}}$, and

- $F \subseteq Q$ is a set of rejecting states.

The automaton is such that it rejects a trace if it violates $\Phi$, i.e., if rejecting states are visited infinitely often. Accordingly, it accepts a concurrent program $\left(P_{1}\left\|P_{2}\right\|\right.$ sched, $\left.v_{0}\right)$ if no trace in $\llbracket P_{1}\left\|P_{2}\right\|$ sched, $v_{0} \rrbracket$ violates $\Phi$. See [18] for more background.

Let $X^{\prime}=X_{1}^{\prime} \cup X_{2}^{\prime}$. We constrain functions $f_{i}$ and $\mu_{i}$ with respect to an additional annotation function $\lambda: Q \times \mathbb{B}^{X^{\prime}} \rightarrow \mathbb{N} \cup\{\perp\}$. In the following, let $\tau_{i}^{\prime}(\bar{x} \circ \bar{z}, \bar{o}, \bar{y})$ denote the combined update function for the original state variables and additional memory variables, explicitly written as

$$
\tau_{i}\left(\bar{x} \circ \bar{z}, \bar{o}, \bar{y} \circ f_{i}(\bar{x}, \bar{z}, \bar{o}, \bar{y})\right) \circ \mu_{i}(\bar{x} \circ \bar{z}, \bar{o}, \bar{y}) .
$$

Similar to the original bounded synthesis encoding [18], we require that

$$
\lambda\left(q_{0}, v_{0} \uparrow_{X^{\prime}}\right) \in \mathbb{N} .
$$

If (1) $\left(q,\left(\bar{x}_{1}, \bar{x}_{2}\right)\right)$ is a composed state with $\lambda\left(q,\left(\bar{x}_{1}, \bar{x}_{2}\right)\right) \in \mathbb{N}$, (2) $\bar{y}_{1} \in \mathbb{B}^{Y_{1}}, \bar{y}_{2} \in \mathbb{B}^{Y_{1}}$ are inputs and $q^{\prime} \in Q$ is a state of the automaton such that there is a transition $\left(q, q^{\prime}\right) \in$ $\Delta$ that is labeled with $\left(\bar{y}_{1}, \bar{y}_{2}\right)$, and (3) $q^{\prime}$ is a non-rejecting state of $\mathcal{U}_{\Phi}$, then we require

$$
\lambda\left(q^{\prime},\left(\tau_{1}^{\prime}\left(\bar{x}_{1}, \bar{o}_{1}, \bar{y}_{1}\right), \tau_{2}^{\prime}\left(\bar{x}_{2}, \bar{o}_{2}, \bar{y}_{2}\right)\right)\right) \geq \lambda\left(q,\left(\bar{x}_{1}, \bar{x}_{2}\right)\right),
$$

where values of $\bar{o}_{1}, \bar{o}_{2}$ are determined by values of $\bar{x}_{2}$ and $\bar{x}_{1}$, respectively (and the subset of states of one process which is observable by the other process).

Finally, if conditions (1) and (2) above hold, and $q^{\prime}$ is rejecting in $\mathcal{U}_{\Phi}$, we require

$$
\lambda\left(q^{\prime},\left(\tau_{1}^{\prime}\left(\bar{x}_{1}, \bar{o}_{1}, \bar{y}_{1}\right), \tau_{2}^{\prime}\left(\bar{x}_{2}, \bar{o}_{2}, \bar{y}_{2}\right)\right)\right)>\lambda\left(q,\left(\bar{x}_{1}, \bar{x}_{2}\right)\right) .
$$

Intuitively, these constraints ensure that in no execution starting from $\left(q_{0}, v_{0}\right)$, the automaton will visit rejecting states infinitely often. Finkbeiner and Schewe [18] have shown that these constraints are satisfiable if and only if there exist implementations of $P_{1}, P_{2}$ with state variables $X_{1}, X_{2}$ that satisfy $\Phi$. With our additional constraints on the original $\tau_{1}, \tau_{2}$ and the integration of the $f_{i}$ and $\mu_{i}$ as new uninterpreted functions, they are satisfiable if there exist $b$-bounded refinements of $P_{1}, P_{2}$ (based on $C_{1}, C_{2}$ ) that 
satisfy $\Phi$. An SMT solver can then be used to find interpretations of the $f_{i}$ and $\mu_{i}$, as well as the auxiliary annotation functions that witness correctness of the refinement.

Correctness. The proposed algorithm for bounded synthesis from program sketches is correct and will eventually find a solution if it exists:

Proposition 1. Any model of the SMT constraints will represent a refinement of the program sketches such that their composition satisfies the specification.

Proposition 2. There exists a model of the SMT constraints if there exist b-bounded refinements $P_{1}^{\prime} \preceq P_{1}, P_{2}^{\prime} \preceq P_{2}$ that satisfy the specification.

Proof ideas for correctness can be found in an extended version [3] of this paper.

Optimization of Solutions. Let cost $: \mathcal{P} \times \mathcal{P} \rightarrow \mathbb{N}$ be a user-defined cost function. We can synthesize an implementation $P_{1}^{\prime}, P_{2}^{\prime} \in \mathcal{P}$ with maximal cost $c$ by adding the constraint $\operatorname{cost}\left(P_{1}^{\prime}, P_{2}^{\prime}\right) \leq c$ (and a definition of the cost function), and we can optimize the solution by searching for implementations with incrementally smaller cost. For instance, a cost function could count the number of memory updates in order to optimize solutions for simplicity.

\subsection{SMT-Based AGS}

Based on the encoding from Section 5.1 this section presents an extension that solves the AGS problem. Recall that the inputs to AGS are two program sketches $P_{1}, P_{2}$ with $P_{i}=\left(X_{i}, O_{i}, Y_{i}, \tau_{i}\right)$, two sets $C_{1}, C_{2}$ of controllable variables with $C_{i} \subseteq Y_{i}$, two specifications $\Phi_{1}, \Phi_{2}$, and a start valuation $v_{0} \in \mathbb{B}^{X \cup Y}$, where $Y=Y_{1} \cup Y_{2}$. The goal is to obtain refinements $P_{1}^{\prime} \preceq P_{1}$ and $P_{2}^{\prime} \preceq P_{2}$ such that:

(i) $\llbracket P_{1}^{\prime}\left\|P_{2}\right\|$ sched, $v_{0} \rrbracket \subseteq\left(\right.$ fair $\left.\wedge \Phi_{2} \rightarrow \Phi_{1}\right)$

(ii) $\llbracket P_{1}\left\|P_{2}^{\prime}\right\|$ sched, $v_{0} \rrbracket \subseteq\left(\right.$ fair $\left.\wedge \Phi_{1} \rightarrow \Phi_{2}\right)$

(iii) $\llbracket P_{1}^{\prime}\left\|P_{2}^{\prime}\right\|$ sched, $v_{0} \rrbracket \subseteq\left(\right.$ fair $\left.\rightarrow \Phi_{1} \wedge \Phi_{2}\right)$.

Using the approach presented above, we can encode each of the three items into a separate set of SMT constraints, using the same function symbols and variable identifiers in all three problems. In more detail, this means that we

1. encode (i), where we ask for a model of $f_{1}$ and $\mu_{1}$ such that $P_{1}^{\prime}$ with $\tau_{1}^{\prime}$ and $P_{2}$ with the given $\tau_{2}$ satisfy the first property,

2. encode (ii), where we ask for a model of $f_{2}$ and $\mu_{2}$ such that $P_{1}$ with the given $\tau_{1}$ and $P_{2}^{\prime}$ with $\tau_{2}^{\prime}$ satisfy the second property, and

3. encode (iii), where we ask for models of $f_{i}$ and $\mu_{i}$ for $i \in\{1,2\}$ such that $P_{1}^{\prime}$ and $P_{2}^{\prime}$ with $\tau_{1}^{\prime}$ and $\tau_{2}^{\prime}$ satisfy the third property.

Then, a solution for the conjunction of all of these constraints must be such that the resulting refinements of $P_{1}$ and $P_{2}$ satisfy all three properties simultaneously, and are thus a solution to the AGS problem. Moreover, a solution to the SMT problem exists if and only if there exists a solution to the AGS problem. 


\subsection{Extensions}

While not covered by the definition of AGS in Section 3, we can easily extend our algorithm to the following cases:

1. If we allow the sets $Z_{1}, Z_{2}$ to be non-disjoint, then the synthesis algorithm can refine processes also by adding shared variables.

2. Also, our algorithms can easily be adapted to AGS with more than 2 processes, as defined in [13].

\section{Experiments}

We implemented 4 our approach as an extension to BoSY, the bounded synthesis backend of the parameterized synthesis tool PARTY [26]. The user defines the sketch in SMT-LIB format with a special naming scheme. The specification is given in LTL. The amount of memory is defined using an integer constant $M$, which is increased until a solution is found. To optimize solutions, the user can assert that some arbitrarily computed cost must be lower than some constant Opt. Our tool will find the minimal value of Opt such that the problem is still realizable. Our tool can also run cooperative cosynthesis and verify existing solutions. Due to space constraints, we can only sketch our experiments here. Details can be found in the extended version [3] of this paper.

For a simple peer-to-peer file sharing protocol [19], we synthesize conditions that define when a process uploads or downloads data. The specification requires that all processes download infinitely often, but a process can only download if the other one uploads. Without AGS, we obtain a brittle solution: if one process is changed to upload and download simultaneously, the other process will starve, i.e., will not download any more. The reason is that cooperative co-synthesis can produce solutions where the correctness of one process relies on a concrete realization of the other processes. With AGS, this problem does not exist. Synthesis takes only one second for this example.

Our next experiment is performed on a double buffering protocol, taken from [40]. There are two buffers. While one is read by $P_{1}$, the other one is written by $P_{2}$. Then, the buffers are swapped. We synthesize waiting conditions such that the two processes can never access the same buffer location simultaneously. The example is parameterized by the size $N$ of the buffers. Table 1 lists the synthesis times for increasing $N$. We use bitvectors to encode the array indices, and observe that the computation time mostly depends on the bitwidth. This explains the jumps whenever $N$ reaches the next power of two.

Table 1. Synthesis times [sec] for increasing $N$.

\begin{tabular}{|l|c|c|c|c|c|c|c|c|c|}
\hline $\mathrm{N}$ & 1 & 2 & 3 & 4 & 5 & 6 & 7 & 8 & 15 \\
\hline AGS & 1 & 5 & 5 & 54 & 51 & 49 & 47 & 1097 & 877 \\
non-AGS & 1 & 4 & 4 & 38 & 35 & 32 & 31 & 636 & 447 \\
\hline
\end{tabular}
Cooperative co-synthesis is only slightly faster than AGS on this example.

Finally, we use our tool to synthesize atomic sections in a simplified version of the i2c Linux kernel driver in order to fix a real bug 5 . This example has been taken

\footnotetext{
${ }^{4}$ Available at/http://www.iaik.tugraz.at/content/research/

design_verification/others

${ }^{5}$ See http://kernel.opensuse.org/cgit/kernel/commit/

?id=7a7d6d9c5fcd4b674da38e814cfc0724c67731b2
} 
from [6]. We synthesize two functions $f_{1}$ and $f_{2}$ that map the program counter value of the respective process to true or false. The value true means that the process cannot be interrupted at this point in the program, i.e., the two adjacent instructions are executed atomically. We also assign costs to active atomic sections, and let our tool minimize the total costs. A meaningful solution with minimal costs is computed in 54 seconds.

\section{Related Work}

Reactive Synthesis. Automatic synthesis of reactive programs from formal specifications, as defined by Church [14], is usually reduced either to games on finite graphs [5], or to the emptiness problem of automata over infinite trees [35]. Pnueli and Rosner [33] proposed synthesis from LTL specifications, and showed its 2EXPTIME complexity based on a doubly exponential translation of the specification into a tree automaton. We use extensions of the game-based approach (see below) to obtain new complexity results for AGS, while our implementation uses an encoding based on tree automata [18] that avoids one exponential blowup compared to the standard approaches [27].

We consider the synthesis of concurrent or distributed reactive systems with partial information, which has been shown to be undecidable in general [34], even for simple safety fragments of temporal logics [38]. Several approaches for distributed synthesis have been proposed, either by restricting the specifications to be local to each process [28], by restricting the communication graph to pipelines and similar structures [17], or by falling back to semi-decision procedures that will eventually find an implementation if one exists, but in general cannot detect unrealizability of a specification [18]. Our synthesis approach is based on the latter, and extends it with synthesis from program sketches [39], as well as the assume-guarantee paradigm [9].

Graph Games. Graph games provide a mathematical foundation to study reactive synthesis problems [14:522]. For the traditional perfect-information setting, the complexity of solving games has been deeply studied; e.g., for reachability and safety objectives the problem is PTIME-complete [23]1; for GR(1) the problem can be solved in polynomial time [32]; and for LTL the problem is 2EXPTIME-complete [33]. For two player partial-information games with reachability objectives, EXPTIME-completeness was established in [36], and symbolic algorithms and strategy construction procedures were studied in [8/2]. However, in the setting of multi-player partial-observation games, the problem is undecidable even for three players [31] and for safety objectives as well [11]. While most of the previous work considers only the general problem and its complexity, the complexity distinction we study for memoryless strategies, and the practical SMT-based approach to solve these games has not been studied before.

Equilibria Notions in Games. In the setting of two-player games for reactive synthesis, the goals of the two players are complementary (i.e., games are zero-sum). For multi-player games there are various notions of equilibria studied for graph games, such as Nash equilibria [29] for graph games that inspired notions of rational synthesis [19]; refinements of Nash equilibria such as secure equilibria [10] that inspired assume-guarantee synthesis (AGS) [9], and doomsday equilibria [7]. An alternative to Nash equilibria and its refinements are approaches based on iterated admissibility [4]. 
Among the various equilibria and synthesis notions, the most relevant one for reactive synthesis is AGS, which is applicable for synthesis of mutual-exclusion protocols [9] as well as for security protocols [13]. The previous work on AGS is severely restricted by perfect information, whereas we consider the problem under the more general framework of partial information, the need of which was already advocated in applications in [24].

\section{Conclusion}

Assume-Guarantee Synthesis (AGS) is particularly suitable for concurrent reactive systems, because none of the synthesized processes relies on the concrete realization of the others. This feature makes a synthesized solution robust against changes in single processes. A major limitation of previous work on AGS was that it assumed perfect information about all processes, which implies that synthesized implementations may use local variables of other processes. In this paper, we resolved this shortcoming by (1) defining AGS in a partial information setting, (2) proving new complexity results for various sub-classes of the problem, (3) presenting a pragmatic synthesis algorithm based on the existing notion of bounded synthesis to solve the problem, (4) providing the first implementation of AGS, which also supports the optimization of solutions with respect to user-defined cost functions, and (5) demonstrating its usefulness by resolving sketches of several concurrent protocols. We believe our contributions can form an important step towards a mixed imperative/declarative programming paradigm for concurrent programs, where the user writes sequential code and the concurrency aspects are taken care of automatically.

In the future, we plan to work on issues such as scalability and usability of our prototype, explore applications for security protocols as mentioned in [24], and research restricted cases where the AGS problem with partial information is decidable.

\section{References}

1. Beeri, C.: On the membership problem for functional and multivalued dependencies in relational databases. ACM Trans. on Database Systems 5, 241-259 (1980)

2. Berwanger, D., Chatterjee, K., De Wulf, M., Doyen, L., Henzinger, T.A.: Strategy construction for parity games with imperfect information. I\& C 208(10), 1206-1220 (2010)

3. Bloem, R., Chatterjee, K., Jacobs, S., Könighofer, R.: Assume-guarantee synthesis for concurrent reactive programs with partial information. CoRR, abs/1411.4604 (2014)

4. Brenguier, R., Raskin, J.F., Sassolas, M.: The complexity of admissibility in omega-regular games. In: CSL-LICS, ACM (2014)

5. Büchi, J.R., Landweber, L.H.: Solving sequential conditions by finite-state strategies. Transactions of the AMS 138, 295-311 (1969)

6. Černý, P., Henzinger, T.A., Radhakrishna, A., Ryzhyk, L., Tarrach, T.: Efficient synthesis for concurrency by semantics-preserving transformations. In: Sharygina, N., Veith, H. (eds.) CAV 2013. LNCS, vol. 8044, pp. 951-967. Springer, Heidelberg (2013)

7. Chatterjee, K., Doyen, L., Filiot, E., Raskin, J.-F.: Doomsday equilibria for omega-regular games. In: McMillan, K.L., Rival, X. (eds.) VMCAI 2014. LNCS, vol. 8318, pp. 78-97. Springer, Heidelberg (2014) 
8. Chatterjee, K., Doyen, L., Henzinger, T.A., Raskin, J.-F.: Algorithms for omega-regular games of incomplete information. In: Logical Methods in Computer Science, vol. 3(3:4) (2007)

9. Chatterjee, K., Henzinger, T.A.: Assume-guarantee synthesis. In: Grumberg, O., Huth, M. (eds.) TACAS 2007. LNCS, vol. 4424, pp. 261-275. Springer, Heidelberg (2007)

10. Chatterjee, K., Henzinger, T.A., Jurdzinski, M.: Games with secure equilibria. Theor. Comput. Sci. 365(1-2), 67-82 (2006)

11. Chatterjee, K., Henzinger, T.A., Otop, J., Pavlogiannis, A.: Distributed synthesis for LTL fragments. In: FMCAD, pp. 18-25. IEEE (2013)

12. Chatterjee, K., Kößler, A., Schmid, U.: Automated analysis of real-time scheduling using graph games. In: HSCC, pp. 163-172. ACM (2013)

13. Chatterjee, K., Raman, V.: Assume-guarantee synthesis for digital contract signing. Formal Asp. Comput. 26(4), 825-859 (2014)

14. Church, A.: Logic, arithmetic, and automata. In: Proceedings of the International Congress of Mathematicians, pp. 23-35 (1962)

15. Clarke, E.M., Grumberg, O., Peled, D.: Model checking. MIT Press (2001)

16. Finkbeiner, B., Jacobs, S.: Lazy synthesis. In: Kuncak, V., Rybalchenko, A. (eds.) VMCAI 2012. LNCS, vol. 7148, pp. 219-234. Springer, Heidelberg (2012)

17. Finkbeiner, B., Schewe, S.: Uniform distributed synthesis. In: LICS, IEEE (2005)

18. Finkbeiner, B., Schewe, S.: Bounded synthesis. STTT 15(5-6), 519-539 (2013)

19. Fisman, D., Kupferman, O., Lustig, Y.: Rational synthesis. In: Esparza, J., Majumdar, R. (eds.) TACAS 2010. LNCS, vol. 6015, pp. 190-204. Springer, Heidelberg (2010)

20. Fortune, S., Hopcroft, J.E., Wyllie, J.: The directed subgraph homeomorphism problem. Theor. Comput. Sci, 111-121 (1980)

21. Grädel, E., Thomas, W., Wilke, T. (eds.): Automata, Logics, and Infinite Games. LNCS, vol. 2500. Springer, Heidelberg (2002)

22. Gurevich, Y., Harrington, L.: Trees, automata, and games. In: STOC, pp. 60-65. ACM (1982)

23. Immerman, N.: Number of quantifiers is better than number of tape cells. J. Comput. Syst. Sci. 22, 384-406 (1981)

24. Jamroga, W., Mauw, S., Melissen, M.: Fairness in non-repudiation protocols. In: Meadows, C., Fernandez-Gago, C. (eds.) STM 2011. LNCS, vol. 7170, pp. 122-139. Springer, Heidelberg (2012)

25. Jobstmann, B., Staber, S., Griesmayer, A., Bloem, R.: Finding and fixing faults. J. Comput. Syst. Sci. 78(2), 441-460 (2012)

26. Khalimov, A., Jacobs, S., Bloem, R.: PARTY parameterized synthesis of token rings. In: Sharygina, N., Veith, H. (eds.) CAV 2013. LNCS, vol. 8044, pp. 928-933. Springer, Heidelberg (2013)

27. Kupferman, O., Vardi, M.Y.: Safraless decision procedures. In: FOCS (2005)

28. Madhusudan, P., Thiagarajan, P.S.: Distributed controller synthesis for local specifications. In: Orejas, F., Spirakis, P.G., van Leeuwen, J. (eds.) ICALP 2001. LNCS, vol. 2076, pp. 396-407. Springer, Heidelberg (2001)

29. Nash, J.F.: Equilibrium points in $n$-person games. Proceedings of the National Academny of Sciences USA 36, 48-49 (1950)

30. Papadimitriou, C.H.: Computational complexity. Addison-Wesley (1994)

31. Peterson, G.L., Reif, J.H.: Multiple-person alternation. In: FOCS. IEEE (1979)

32. Piterman, N., Pnueli, A., Sa'ar, Y.: Synthesis of reactive(1) designs. In: Emerson, E.A., Namjoshi, K.S. (eds.) VMCAI 2006. LNCS, vol. 3855, pp. 364-380. Springer, Heidelberg (2006)

33. Pnueli, A., Rosner, R.: On the synthesis of a reactive module. In: POPL (1989)

34. Pnueli, A., Rosner, R.: Distributed reactive systems are hard to synthesize. In: FOCS, pp. 746-757. IEEE (1990) 
35. Rabin, M.O.: Automata on Infinite Objects and Churchs Problem. American Mathematical Society (1972)

36. Reif, J.H.: The complexity of two-player games of incomplete information. J. Comput. Syst. Sci. 29(2), 274-301 (1984)

37. Savitch, W.J.: Relationships between nondeterministic and deterministic tape complexities. JCSS 4(2), 177-192 (1970)

38. Schewe, S.: Distributed synthesis is simply undecidable. IPL 114(4), 203-207 (2014)

39. Solar-Lezama, A.: Program sketching. STTT 15(5-6), 475-495 (2013)

40. Vechev, M.T., Yahav, E., Yorsh, G.: Abstraction-guided synthesis of synchronization. In: POPL, pp. 327-338. ACM (2010) 